Brief note

\title{
A SYNTHESIS MODEL FOR FORCING ACTION ARRANGEMENT IN THE SYSTEM OF REDUCING DYNAMIC LOADS OF A MOBILE MACHINE
}

\author{
H. KAŹMIERCZAK*, T. PAWŁOWSKI and K. ZEMBROWSKI \\ Industrial Institute of Agricultural Engineering \\ Starołęcka 31, 60-963 Poznań, POLAND \\ E-mail: kazmhenr@pimr.poznan.pl
}

\begin{abstract}
An idea is presented for a method to lower excessive dynamic loads in the system of supporting structure, mechanical-hydraulic forcing system, vibration isolation system, protective unit. The dynamic characteristics of the system are determined by the method of dynamic susceptibility. An analytical model of the system was built (mobile machine to carry out protective treatments; project WDN-POIG.01.03.01-00-164/09).
\end{abstract}

Key words: structural changes, dynamic susceptibility, forcing system model, dynamic mobility.

\section{Introduction}

Growing requirements concerning durability and reliability of machines result from a growth of their operating parameters. Therefore, along the growth of modern research methods, intense evaluations are run of the effort state of structures under their operational conditions, taking into account an analysis of constructional material degradation process. Durability and strength of a mechanical system (system component) are determined by a number of factors, e.g., load type and amplitude, space and time characteristics of such load, corrosion and ageing process, material strength properties, etc. Hence, there is a need to formulate holistic criteria of dynamic effort loads on constructions and energetic methods to establish fatigue life of system components and formulate an energetic model of changes in mechanical properties of a material. Machine components are subjected to bending, torsional and static loads (weight and preload) as they are linked to the functions served by the machine. These loads cause fatigue processes in the construction materials in points of stress accumulation (Kaźmierczak, 2013). Stresses occurring in a machine component result from the course of a forcing action which most often is of a stochastic nature of a spectrum similar to coloured noise in frequency bands in which forcing actions occur from forces of a periodical course, such as centrifugal forces or forces coming from masses in a periodical reciprocating motion. These stresses are a function of the dynamics of the entire complex mechanical system. Material fatigue failures and wear causing changes in the coefficients of friction and looseness in connections are a cause of parameter changes in a dynamic system, such as susceptibility or damping, which influences the course of stresses. As a result, despite the low level of a medium stress there is always a possibility in a machine component to exceed the threshold value of stress causing material failures in the form of micro-crack propagation (Kaźmierczak et al., 2012). An overview of various defect measures, described in scientific literature, was presented by Yang and Fatami (1998). Changes in the dynamic condition of a mechanical object, described by energetic characteristics of vibration loads on nodes of a mechanical system, are an important indicator of differences or changes occurring in its structure. Research into imaginary parts and real parts of the testing force powers in the frequency function, enables determination of changes (maxima) in these functions. The shift of characteristics extremes and the occurrence of minima (anti-resonance) in energetic characteristics of dynamic rigidity constitutes key information about preliminary tensions applied

\footnotetext{
* To whom correspondence should be addressed
} 
in the object or information on degradation state of a mechanical object which manifests itself in dynamic rigidity changes. As previous research shows, changeable powers of vibration damping forces, being a determinant of mechanical energy dissipation, is a value determining structural degradation of a mechanical object throughout duration of the process. Intense changes in dynamic rigidity manifesting themselves in cracking, occur mainly in the final phase of technical degradation of a mechanical object. An analysis of those changes will make it possible to determine boundary values of load powers which trigger the initiation of structural degradation processes (e.g. cracking) in a mechanical object component.

\section{Modelling of the machine technical condition}

Dynamic properties of a machine are a derivative of the spatial distribution of the machine's physical parameters and edge conditions and also the way of its aggregation with the drive system. Dynamic properties of a machine should be assessed in a close relation to forcing action parameters (loads). The following technical parameters should be taken into account in an assessment of a machine: machine mass, mass ratio between the machine and the moving component, type of supporting structure (suspension), etc. The overall requirement is the following: in each and every construction, the maximum reduction of exciting forces should be pursued. The most frequent reasons for the occurrence of large exciting forces are static unbalance of impellers, dynamic unbalance of drive shafts or impellers in the technology systems of engineering machines, component misalignment in the driving-working system, occurrences of looseness, dissymmetry in power transmission systems, assembly errors, motion of the operating medium in the machine. Dynamic properties of a machine being a complex system are determined by dynamic properties of its units (components) and methods of their connection. The machine should thus be viewed in the aggregate with the supporting structure, as the dynamic properties of the supporting structure determine the machine behaviour during the occurrence of exciting forces equally to the properties of the machine itself. The machine (a complex mechanical system) should basically be treated as a system which has an indefinite quantity of free vibration frequencies. However, it can be viewed that, in most structural components, particularly in heavy machinery and engineering machines, only slight relative strains occur and, as a result, these components behave almost as rigid bodies. Therefore, in the machine calculation model the system is subjected to a reduction (reduction of the degrees of freedom in the model), and the machine is thereafter treated as a system of a limited amount of freedom degrees.

For the assessment of the technical condition and durability of a machine subjected to a multi-input vector of external forcing actions, the energetic method of dynamic load power distribution is applied, as the processes determining the durability profile of an object have an energetic dimension.

The input power of a mechanical system at a given time $t$ equals

$$
F(t) \cdot v(t)
$$

where: $F(t)$ represents force measured at the point of excitation of the structure, and $v(t)$ represents spot speed at the point of excitation.

The output power, averaged out over time, is described by the following expression

$$
N_{\text {in }}=\frac{1}{T} \cdot \int_{0}^{T} F(t) \cdot v(t) d t,
$$

and in the frequency domain

$$
N_{i n}=\left[\int_{0}^{\infty} G_{v F}(\omega) d \omega\right]
$$


where: $G_{v F}(\omega)$ represents one-sided cross-spectrum of the force and velocity of vibrations at the point of excitation. As acceleration sensors are mainly used to measure vibrations, vibration velocities are represented by accelerations

$$
N_{\text {in }}=\frac{1}{\omega} \cdot\left[\int_{0}^{\infty} G_{a F}(\omega) d \omega\right]
$$

or in specific frequency bands

$$
N_{\omega_{1}, \omega_{2}}=\frac{1}{\omega} \cdot\left[\int_{\omega_{2}}^{\omega_{1}} G_{a F}(\omega) d \omega\right] .
$$

Input power measurements require the real and imaginary parts of the cross-spectrum $G_{a F}(f)$ to be calculated. Hence, unlike in energy measurements, where phase information is not significant, in the case of input power measurement it is necessary to know the force and acceleration phase at the point of excitation.

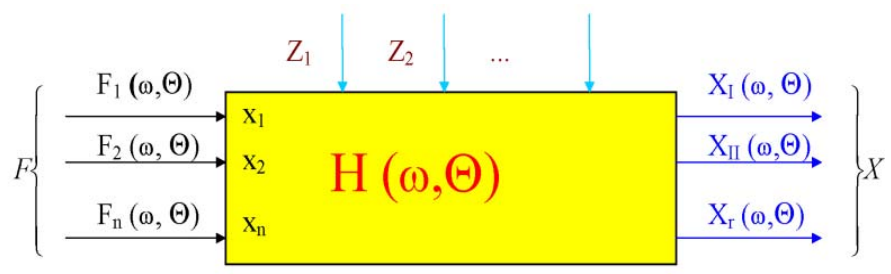

Fig.1. Model of machine (Kaźmierczak, 2001).

A holistic model of load changes in the process of mechanical system degradation is described by the matrix of spectral densities of dynamic load powers (multi input-multi output system), (Fig.1, Kaźmierczak, 2001)

$$
\left\{G_{N_{i k}}(j \omega, \Theta)\right\}=\boldsymbol{H}_{V_{i k}}(j \omega, \Theta) \cdot \boldsymbol{G}_{F_{k} F_{k}}(j \omega, \Theta)
$$

where: $\boldsymbol{H}_{V_{i k}}-$ dynamic mobility matrix,

$\boldsymbol{G}_{F F-}$ external forcing actions spectral density matrix,

$k$ - application points of external forcing actions,

$i$ - points of discrete stress tensor model.

By synthesizing matrix elements of power spectral densities of load powers (1), the load power matrix $N(\Theta)$ can be achieved for the system, which is a space-time profile of system degradation.

A spatial profile of system effort condition described holistically in the function of time $\Theta$ can be defined in the form of a matrix of energetic efforts set at points " $i$ " of their intellectual crossing by mutually perpendicular planes. Efforts come from external forcing powers set at points " $k$ " of the system (Kaźmierczak, 2009)

$$
\overline{\boldsymbol{W}}_{i k}(\Theta)=\left\{\begin{array}{llll}
W_{11} & W_{12} & \ldots & W_{1 n} \\
W_{21} & W_{22} & \ldots & W_{2 n} \\
\hline W_{r 1} & W_{r 2} & \ldots & W_{r n}
\end{array}\right\}
$$


where $k=1,2, \ldots . n$-application points of external forcing actions $i=1,2, \ldots, n, n+1, n+2, \ldots r$ - effort test points.

In the single-input single-output system, the energetic model described by the matrices 1-4 reduces to one element. Each element of the matrix of energetic efforts of structure components may be defined in the following way

$$
\bar{W}_{i k}=\frac{\int \bar{N}_{i k}(\Theta) d \Theta}{S_{i}}
$$

where: $S_{i}$ - cross section area of object component (material sample) at point " $i$ ", $\bar{N}_{i k}$ are average load powers determined at points " $i$ " as a result of forcing action applications at points " $k$ ".

\section{Determination of machine dynamic characteristics by the method of the modal analysis}

In the frequency domain, the vector of dislocation $X_{i}(\omega, \Theta)$ is described by the following equation

$$
\boldsymbol{X}_{i}(\omega, \Theta)=\boldsymbol{H}_{i k}(j \omega, \Theta) \boldsymbol{F}_{k}(\omega, \Theta)
$$

while

$$
\boldsymbol{H}(j \omega, \Theta)=\left[\boldsymbol{K}-\omega^{2} \boldsymbol{M}+j \omega \boldsymbol{C}\right]^{-1}
$$

where:

$\boldsymbol{M}, \boldsymbol{C}, \boldsymbol{K}-$ mass, damping and rigidity matrices

$\boldsymbol{X}, \dot{\boldsymbol{X}}, \ddot{\boldsymbol{X}}-$ dislocation, velocity and rigidity vectors

$\boldsymbol{F}(t)$ - forcing vector.

Dynamic mobility is defined as the ratio of vibration velocity at point $i$ of the machine to exciting force set at point $\boldsymbol{k}$.

$$
H_{V_{i k}}(j \omega)=\frac{V_{i}(\omega)}{F_{k}(\omega)} .
$$

Considering a minimization of errors in the process of identification, these characteristics are established through spectral densities

$$
H(j \omega)=\frac{G_{X F}(j \omega)}{G_{F F}(j \omega)} .
$$

The method of modal analysis is applied to determine, respectively, free vibration frequencies $\omega_{0_{i}}$, dynamic susceptibility modules $\left|H_{i k}(j \omega)\right|$, and their respective real and imaginary parts, $\operatorname{Re} H_{i k}$ and $\operatorname{Im} H_{i k}$. 


\section{An analytical model of rotor mechanical-hydraulic system}

The key quantity describing the driving -working system of a rotor machine is the torque moment $M_{\mathrm{s}}$ transmitted in rotary motion by a drive-working shaft.

Quantities describing the dynamic properties of the unit are hydraulic-mechanical mobility and impedance. Mobility of the rotor driving-working system, being the function of mechanical-hydraulic load transmission

$$
H_{p}(j \omega)=\frac{p(\omega)}{F(\omega)}
$$

where:

$p$ - pressure in the pump hydraulic system,

$F$ - component of force tangential to the unit drive shaft,

$\omega$ - vibration frequency.

Mobility of the rotor driving-working system is a complex quantity

$$
H_{p}(j \omega)=\operatorname{Re} H_{p}(\omega)+j \operatorname{Im} H_{p}(\omega) .
$$

Impedance of the rotor driving-working system

$$
R_{p}(j \omega)=\frac{F(\omega)}{p(\omega)}
$$

while

$$
R_{p}(j \omega)=\operatorname{Re} R_{p}(\omega)+j \operatorname{Im} R_{p}(\omega) .
$$

Mobility of the rotor driving-working system (Fig.2, Bishop et al., 2011)

$$
H_{42}=\omega \frac{\alpha_{33} \varepsilon_{33}}{\alpha_{33}+\varepsilon_{33}}
$$

where $\quad \alpha_{23}=\frac{\xi_{22} \varphi_{23}}{\xi_{22}+\varphi_{23}}, \quad \alpha_{33}=\frac{\varphi_{23}\left(\xi_{22}+\varphi_{22}\right)-\varphi_{23}^{2}}{\xi_{22}+\varphi_{23}}, \quad \varphi_{23}=\frac{-1}{I_{2} \omega^{2}}$

$$
\begin{aligned}
& \xi_{22}=\frac{1}{k_{1}-I_{1} \omega^{2}+i c_{1} \omega}, \quad \varphi_{22}=\frac{-1}{I_{2} \omega^{2}}+\frac{1}{k_{2}+i c_{2} \omega}, \\
& \varepsilon_{33}=\frac{k_{3}-I_{3} \omega^{2}+i c_{3} \omega}{I_{3} \omega^{2}\left(k_{3}+i c_{3} \omega\right)+\left(k_{4}+i c_{4} \omega\right)\left[\left(k_{3}-I_{3} \omega^{2}\right)+i c_{3} \omega\right]} .
\end{aligned}
$$

The parameters of the analytical model of rotor driving - working system (Fig.2, Bishop et al., 1972):

- supporting structure $\left(k_{1}, c_{1}\right)$, - powertrain engine flywheel $\left(I_{1}\right)$, - shaft $\left(k_{2}, c_{2}\right)$,

- clutch $\left(I_{2}\right)$,- oil pump shaft $\left(k_{3}, c_{3}\right)$, - impeller $\left(I_{3}\right)$. 


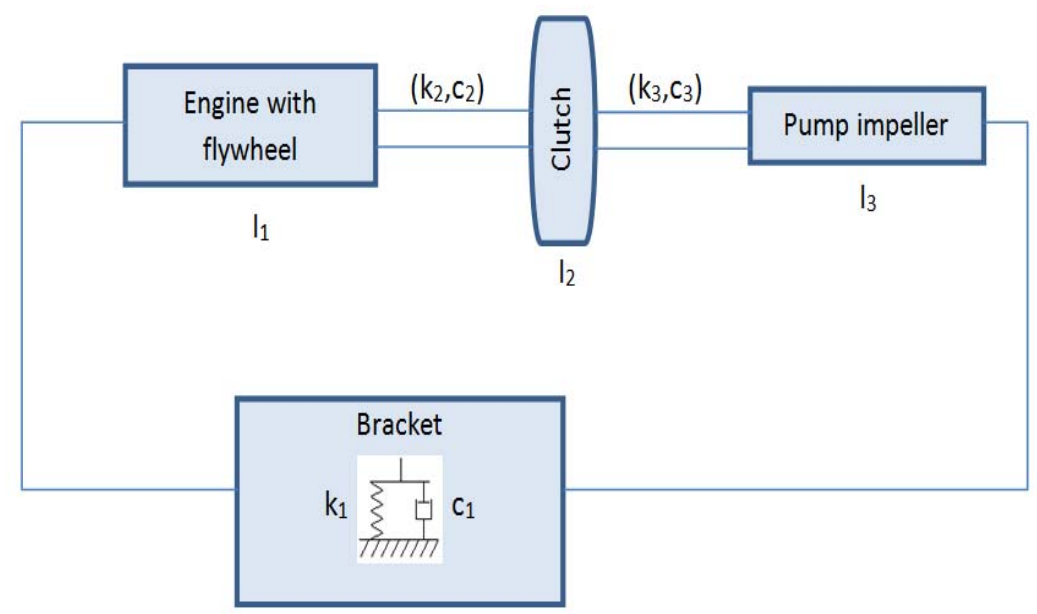

Fig.2. Model of mechanical-hydraulic rotor unit.

In order to determine mobility $H_{41}$ a computer programme was developed, and the dynamic mobility module and the real and imaginary parts were determined.

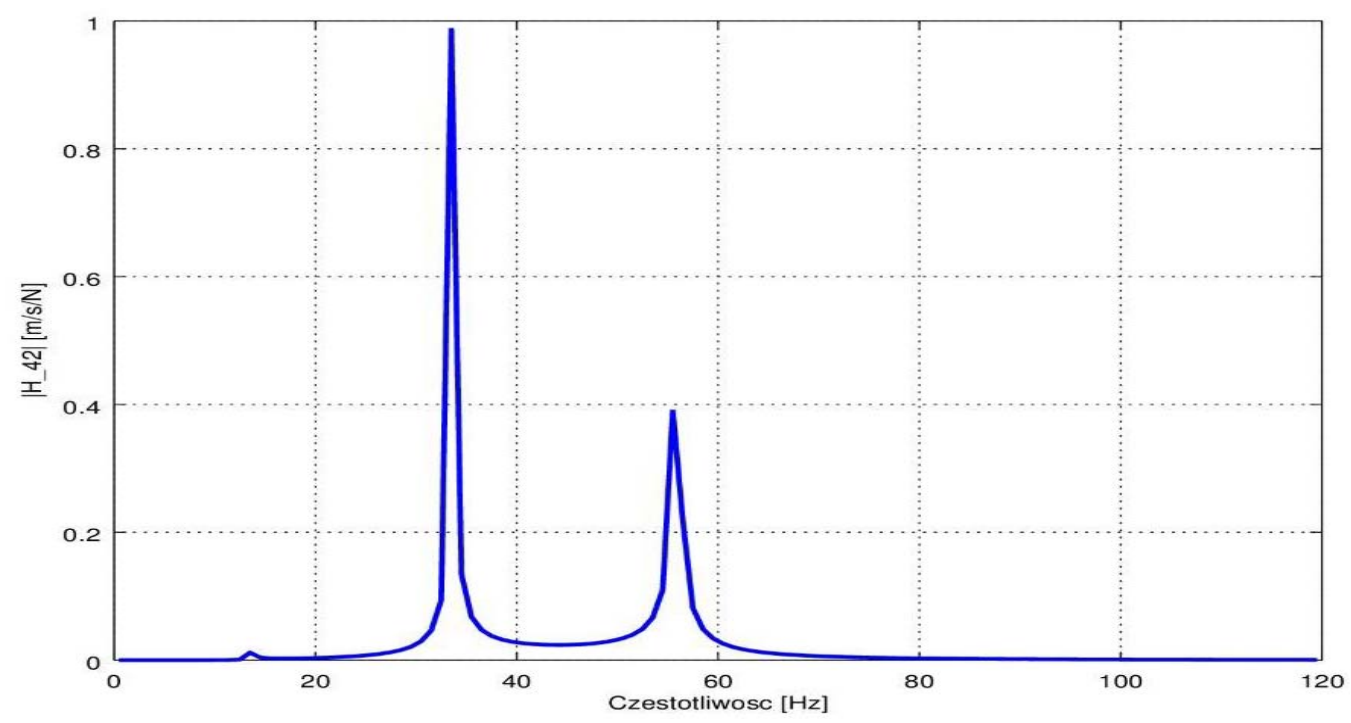

Fig.3. Dynamic mobility module $\mathrm{H}_{42}$ (f).

Figure 3 presents a module example of dynamic mobility $\mathrm{H}_{42}$ (f) of the system model [I1, I2, I3, k1, $\mathrm{k} 2, \mathrm{k} 3, \mathrm{c} 1, \mathrm{c} 2, \mathrm{c} 3$ ], and Fig.4 shows the imaginary part (damping curve) of dynamic mobility in the band of 0.5-120 Hz. Amplitude measurements of the parameters (e.g., damping) will be the key component of the system identification procedure.

In the procedure to optimize the forcing action arrangement, the characteristics of an analytical model will be compared to diagrams determined by experiment and thereafter compared to forcing action frequencies. 


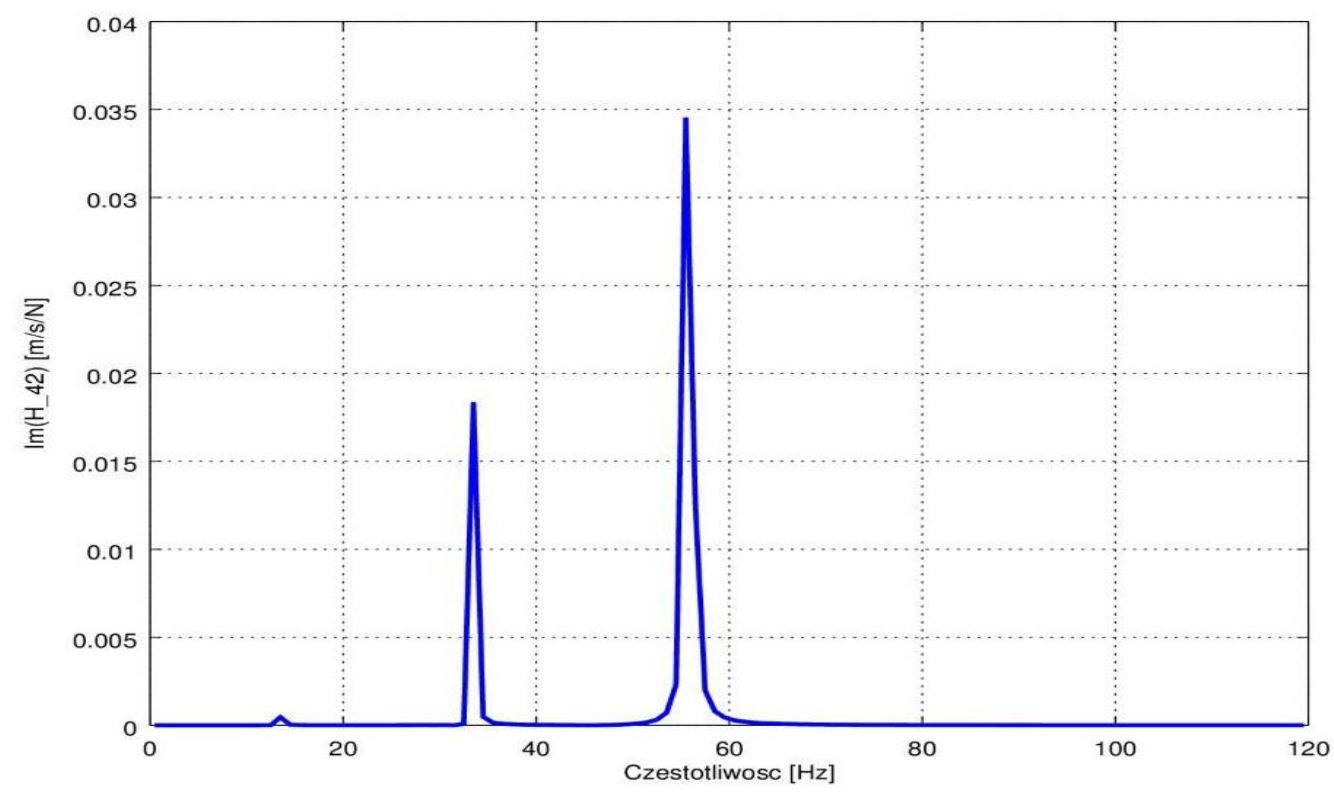

Fig.4. Dynamic mobility imaginary part $\mathrm{ImH}_{42}$ (f).

Based on experimentally determined parameters of operational loads of a machine, an energetic load model will be developed (Formula 1).

\section{Conclusions}

1. The dynamic characteristics of units are the basis for construction optimization.

2. Dynamic properties of a machine being a complex system are determined by dynamic properties of its units and methods of their connection.

3. Quantities describing the dynamic properties of the driving-working system are hydraulic-mechanical mobility and impedance.

4. The optimization works of the solution were carried out, reducing the motion resistance in the system.

\section{References}

Bishop R.E.D. and Johnson D. (2011): The Mechanics of Vibrations. - Cambridge University Press.

Bishop R.E.D., Gladwell G.M.L. and Michaelson S. (1972): Macierzowa analiza drgań. - Warsaw: WNT.

Kaźmierczak H. (2001): Analysis of dynamic load power distribution in mechanical systems. - Publishing House of Poznań University of Technology, Studies 363.

Kaźmierczak H. (2013): Energetyczne Charakterystyki Dynamicznej Degradacji Struktur Mechanicznych. Wydawnictwo PIMR Poznań, ISBN 978-82-927505-7-4.

Kaźmierczak H. and Pawłowski T. (2012): Energetic characteristics degradation of mechanical structures. International Journal of Applied Mechanics and Engineering, vol.17, No.3, pp.861-866.

Kaźmierczak H., Pawłowski T. and Wojniłowicz Ł. (2013): Quantifiable measures of the structural degradation of construction materials. - Diagnostyka. Applied Structural Health, Usage and Condition Monitoring, vol.14, No.4, pp.77-83.

Yang L. and Fatemi A. (1998): Cumulative Fatigue Damage Mechanisms and Quantifying Parameters, A Literature Review. - Testing and Evaluation. 